\title{
A prospective study on maternal and perinatal outcome of gestational diabetes mellitus
}

\author{
Sathiamma P. K.*, Lalithambica Karunakaran
}

Department of Obstetrics and Gynecology, Government TD Medical College, Alappuzha, Kerala, India

Received: 16 April 2017

Accepted: 18 May 2017

\section{*Correspondence:}

Dr. Sathiamma P. K.,

E-mail: sathiammapk@ymail.com

Copyright: $\odot$ the author(s), publisher and licensee Medip Academy. This is an open-access article distributed under the terms of the Creative Commons Attribution Non-Commercial License, which permits unrestricted non-commercial use, distribution, and reproduction in any medium, provided the original work is properly cited.

\section{ABSTRACT}

Background: To identify risk factors, study maternal, fetal and perinatal outcome of pregnancy in relation to the glycemic control and different modalities of management in pregnancy complicated by Gestational Diabetes Mellitus (GDM).

Methods: Descriptive study conducted in Department of OBG, Government TD Medical College Alappuzha, during the one year period January 2005 to December 2005. Study group comprised of 134 women who are diagnosed to have GDM. The aim was to study the maternal, fetal and perinatal outcome.

Results: Despite early diagnosis and treatment the GDM patients in the present study had a statistically significant higher incidence of pregnancy induced hypertension (11.9\%) induction of labor about (37.2\%), caesarean section (58.96\%) preterm delivery (2.99\%) macrosomia (2.9\%). Incidence of perinatal morbidity was $29.8 \%$, common causes being neonatal hypoglycemia (32.5\%), hyperbilirubinemia (12.5\%) meconium aspiration syndrome (7.5\%). Admission to neonatal unit required in (25\%). There were 2 cases of intrauterine deaths, 2 cases of neonatal deaths and no still births. Perinatal mortality was $2.9 \%$.

Conclusions: The occurrence of GDM is a high-risk situation. Maternal morbidity, perinatal morbidity and mortality are increased in women with GDM. All pregnant women should be screened for GDM with a 50gm oral glucose load followed by a glucose determination 1 hour later. This helps to detect all GDM cases earlier and so timely intervention can be done which will reduces the complications. Proper management of GDM during the antenatal period will improve pregnancy outcome.

Keywords: Gestational diabetes mellitus, Macrosomia, Maternal outcome, Perinatal outcome

\section{INTRODUCTION}

GDM is the most common metabolic disorder complicating pregnancy. About 3-5 percent of pregnancies are complicated by diabetes, $90 \%$ of these are GDM the rest being pregestational. GDM is defined as carbohydrate intolerance of variable severity with onset or first recognition during pregnancy. ${ }^{1}$ Some women with GDM have previously unrecognized overt diabetes mellitus (DM). ${ }^{2}$ GDM develops in the latter half of pregnancy and sugar levels return to normal after delivery. If diabetes is not detected early and treated properly it can adversely affect both the mother and fetus.

The prevalence of GDM range from 1-14\% depending on the population and the diagnostic tests employed. Incidence of GDM is increasing due to lack of exercise and changing dietary habits. The disturbed carbohydrate metabolism creates maternal complications like abortions, pregnancy induced hypertension (PIH), polyhydramnios, preterm labor, fetal growth restriction (FGR) recurrent urinary infections (UTI) vulvitis, 
increased incidence of operative delivery and maternal morbidity. Fetal and neonatal complications include fetal malformation, macrosomia perinatal morbidity like prematurity, respiratory distress syndrome (RDS), birth trauma, neonatal hypoglycemia, hypocalcemia and hyperbilirubinemia perinatal morbidity like intrauterine death (IUD) stillbirth (SB) and neonatal death (NND). Longterm adverse effects in the mother include development of Type II DM, hypertension, cardiovascular disease and recurrence in next pregnancy. Long term effects in child include obesity and development of Type II DM. So, it is important rule out GDM in all pregnancies. If selective screening of high risk group alone is done, many cases may be missed. An oral glucose challenge test (OGCT) with $50 \mathrm{gm}$ oral glucose load followed by a glucose determination 1 hour later is an ideal one with sensitivity and specificity of about $90 \%$.

Proper control of DM preconceptionally reduces the incidence of fetal anomalies and glycemic control during the latter half of pregnancy reduces perinatal complications. Intensive glycemic control, fetal surveillance prevention of obstetric complications, timely delivery and intensive neonatal care by a team approach by Obstetrician, Physician preferably Diabetologist and the Neonatologist became the corner stone of management of diabetes in pregnancy. Euglycemia means glucose levels are at target level throughout the whole day which can be achieved by medical nutrition therapy (MNT) insulin therapy, exercise, and fetal and maternal monitoring. MNT means diet that provides the caloric and nutrient needs to sustain pregnancy and normoglycemia. Human insulin is the preferred one and oral hypoglycemic drugs are rarely used Decision to delivery is based on degree of diabetes control, risk factors and fetal well-being, not allowed to go beyond dates even in well-controlled diabetes especially pregestational DM where complications are more.

The objective of the study was to identify the risk factors, study the outcome in relation to glycemic control and the maternal and perinatal outcome in GDM.

\section{METHODS}

This study was conducted in OBG Dept Government TD Medical College Alappuzha during a period of one year from January 2005 to December 2005.Total deliveries during that period was 5647 of which 134 cases had GDM. All gravidas of gestational age between 28 and 40 yrs were included and samples being from the outpatient department, antenatal wards, labor room, postnatal wards, pediatric wards and newborn intensive care unit (NICU). The pregnant women were subjected to an OGCT at 2428 weeks gestation and if the value is $>=130 \mathrm{mg} \%, 100$ gm oral glucose tolerance test (OGTT) done, where fasting, 1, 2 and $3 \mathrm{hr}$ sugar values estimated. One abnormal value was considered as impaired glucose tolerance (IGT) and if two or more values abnormal as
GDM. Patients with GDM were advised MNT, blood sugar checked after 12-14days. Euglycemia is Fasting blood sugar (FBS) $<90 \mathrm{mg} / \mathrm{dl}$ and 2hour Postprandial blood sugar (PPBS) $<120 \mathrm{mg} / \mathrm{dl}$ and if fails to achieve this target level Human insulin started. Insulin dose adjusted according to FBS, $2 \mathrm{hr}$ PPBS level checking every 3 days thereafter weekly if well controlled. The GDM patients are managed in co-operation with Diabetologist, and neonatologist. This has improved the metabolic control as well as fetal salvage rate

In addition to routine investigations and blood sugar estimation, renal and liver function test, platelet count estimation done to assess the mother and serial USS along with other antepartum fetal surveillance tests done to assess the fetus. The fetal well-being was assessed from 28-30 weeks onwards. Doppler ultrasound was done only in selected cases. Earlier admission was done if there is any maternal or fetal compromise. Uncomplicated cases are allowed to go into spontaneous labor or wait till date. Termination is done early only in poorly controlled GDM and at times of fetal jeopardy. Labor was induced at 38 weeks if she was on insulin. Normoglycemia maintained during labor also. Close monitoring of mother and fetus was done during labor and use of partogram helped avoidance of prolongation of labor.

The maternal variables analyzed were maternal age at delivery, gravidity and parity, previous obstetric history, maternal complications, mode of delivery, indications of cesarean and postpartum complications. In the postpartum period insulin was given only after blood sugar estimation. Newborn status was evaluated by apgar scores, observed for anomalies, macrosomia development of hypoglycemia, respiratory distress, sepsis hypocalcemia, hyperbilirubinaemia. H/O shoulder dystocia, admission to NICU, percentage of large for gestational age preterm babies $<37$ weeks, all are studied.

\section{RESULTS}

Out of the 5467 deliveries during the period of study 134 $(2.4 \%)$ cases had GDM. Majority was between 25 and 29 years with a mean of 27.79 years (Table 1 ).

Table 1: Distribution according to age.

\begin{tabular}{|lll|}
\hline Age in years & Number & $\%$ \\
\hline$<-20$ & 0 & 0.0 \\
\hline $21-24$ & 36 & 26.9 \\
\hline $25-29$ & 57 & 42.5 \\
\hline $30-34$ & 30 & 22.4 \\
\hline $35-39$ & 8 & 6.0 \\
\hline $40->$ & 3 & 2.2 \\
\hline Total & 134 & 100 \\
\hline
\end{tabular}

About $40 \%$ belonged to middle income group and $96 \%$ had high school or above level education.32.08\% were primi gravidas and $67.92 \%$ were multi gravid. No history 
of previous pregnancy complications was there in $58.3 \%$ case and the rest had complications like GDM, abortions, $\mathrm{PIH}$, previous macrosomia NND, IUD and congenital anomalies. Normoglycemia was achieved with diet alone in $44(32.8 \%)$ diet plus insulin in $87(64.92 \%)$. Along with insulin metformin also was used in 3 cases.

Table 2: Distribution according to present obstetric complications.

\begin{tabular}{|lll|}
\hline Obstetric complications & Number & $\%$ \\
\hline PIH & 16 & 11.9 \\
\hline PROM & 10 & 7.4 \\
\hline Vulvitis & 24 & 17.9 \\
\hline IUGR & 2 & 1.6 \\
\hline UTI & 34 & 25.4 \\
\hline Hydramnios & 13 & 9.7 \\
\hline Macrosomia & 4 & 2.9 \\
\hline IUD & 2 & 1.6 \\
\hline NND & 2 & 1.6 \\
\hline No complication & 29 & 21.6 \\
\hline
\end{tabular}

Table 3: Distribution according to period of induction.

\begin{tabular}{|lll|}
\hline Period of induction & Number & $\%$ \\
\hline$<36$ Weeks & 2 & 1.49 \\
\hline 36 Weeks & 4 & 2.99 \\
\hline 37 Weeks & 16 & 11.94 \\
\hline 38 Weeks & 90 & 67.16 \\
\hline 39 Weeks & 22 & 16.42 \\
\hline 40 Weeks & Nil & 0 \\
\hline
\end{tabular}

No maternal complication was found in $21.6 \%$ cases Common complications met in this study were PIH 16 cases, hydramnios in 13, UTI in 34 cases, premature rupture of membranes (PROM) was $7.4 \%$ and vulvitis about $17.9 \%$ of cases. Macrosomia was present in $2.9 \%$ of cases and IUD 1.6\% and NND 1.6\%. (Table 2) Normal sonological findings were found in $110(82.08 \%)$ of cases.

Table 4: Mode of delivery.

\begin{tabular}{|lll|}
\hline Mode of delivery & Number & $\%$ \\
\hline FTND & 41 & 30.59 \\
\hline Preterm delivery & 4 & 2.99 \\
\hline Vacuum Extraction & 6 & 4.48 \\
\hline Outlet Forceps & 2 & 1.49 \\
\hline LSCS & 79 & 58.96 \\
\hline IUD Expulsion & 2 & 1.49 \\
\hline
\end{tabular}

Abnormal findings included hydramnios in 11(9.71\%), oligamnios in $2(1.49 \%)$ and Macrosomia $4(2.9 \%)$ congenital anomalies in $5(3.73 \%)$. Regarding duration of pregnancy, 33 cases $(24.6 \%)$ delivered preterm and 101 $(75.4 \%)$ of patients had term delivery $62.68 \%$ patients had spontaneous labor and elective termination was done in $37.32 \%$ of case. In 90 cases $(67.16 \%)$ labor induced around 38 weeks, $16(11.94 \%)$ at 37 wks and before $<=36$ wks for $6(4.4 \%)$ due to complications like PIH and IUGR. No patients were allowed to go past date. (Table 3 ) Out of 55 cases of vaginal delivery, $35.07 \%$ were normal and 8 cases $5.8 \%$ were instrumental. LSCS done for $58.96 \%$ cases, common indications being previous caesarean section, cephalopelvic disproportion, preeclampsia, FGR with failed induction, and failed induction (Table 4). (30.5\%) patients had various complications during labor. PIH 16 (39.03\%) and PROM $10(24.9 \%)$ being the common complications. There were 2 cases of impending eclampsia. Meconium stained amniotic fluid was present in 7 and fetal distress in 5 cases (Table 5).

Table 5: Distribution according to type of labour complications.

\begin{tabular}{|lll|}
\hline Complication & Number & $\%$ \\
\hline PROM & 10 & 24.39 \\
\hline PIH & 16 & 39.03 \\
\hline Impending Eclampsia & 2 & 4.88 \\
\hline Labour Complications & 1 & 2.44 \\
\hline Meconium stained Amniotic fluid & 7 & 17.07 \\
\hline Fetal Distress & 5 & 12.19 \\
\hline
\end{tabular}

The mean birth weight in the study group was $2.9 \mathrm{~kg}$. Two had weight $>4 \mathrm{~kg}$ and 28 babies $(20.8 \%)$ had weight $<2.5 \mathrm{~kg}$ (Table 6).

Table 6: Distribution according to birth weight.

\begin{tabular}{|lll|}
\hline Birth weight in Kg & Number & $\%$ \\
\hline$\leq 2$ & 1 & 0.75 \\
\hline $2-2.4$ & 27 & 20.15 \\
\hline $2.5-2.9$ & 55 & 41.04 \\
\hline $3-3.4$ & 40 & 29.85 \\
\hline $3.5-3.9$ & 9 & 6.72 \\
\hline$\geq 4$ & 2 & 1.49 \\
\hline
\end{tabular}

Table 7: Distribution according to perinatal morbidity.

\begin{tabular}{|lll|}
\hline Type of perinatal morbidity & Number & $\%$ \\
\hline Hypoglycemia & 13 & 32.5 \\
\hline Sepsis & 9 & 22.5 \\
\hline Hyperbilirubinaemia & 5 & 12.5 \\
\hline Poor sucking & 5 & 12.5 \\
\hline Intra cranial hemorrhage & 2 & 5 \\
\hline Meconium aspiration syndrome & 3 & 7.5 \\
\hline TTNB & 2 & 5 \\
\hline Erb's palsy & 1 & 2.5 \\
\hline
\end{tabular}

Regarding perinatal morbidity in 47 (35.07\%) babies, hypoglycemia was the most common one, observed in 13 $(32.5 \%)$ babies. Others were sepsis in $9(22.5 \%)$ babies. Hyperbilirubinaemia was found in $5(12.5 \%)$ babies and meconium aspiration syndrome in $3(7.5 \%)$ babies. Others included intracranial haemorrhage poor sucking, Transient Tachypnoea of New Born (TTNB), Erbs palsy 
(Table 7). There were 4 cases of mortality 2 cases of IUDs and 2 cases of neonatal deaths and no still birth. Of the 2 NNDs 1 was preterm baby with sepsis and 1 baby had intra cranial hemorrhage with septic shock (Table 8).

Table 8: Distribution according to perinatal mortality.

\begin{tabular}{|lll|}
\hline Type of perinatal mortality & Number & $\%$ \\
\hline IUD & 2 & 50 \\
\hline NND & 2 & 50 \\
\hline Still birth & Nil & 0 \\
\hline
\end{tabular}

$3425 \%$ need new born nursery admission due to complications like jaundice, hypoglycemia and sepsis. 58 $43 \%$ babies were observed in new born nursery, as they are large for gestational age babies. Out of 134 GDM babies, $8(5.9 \%)$ babies had congenital Anomalies. 4 babies had congenital heart disease of which two were patent ductus arteriosus. There was 1 case of cleft lip, 1 cleft palate, 1 case of meningomyelocele and 1 case of trachea-esophageal fistula (Table 9).

Table 9: Distribution of congenital anomalies.

\begin{tabular}{|lll|}
\hline Type of Congenital anomalies & Number & $\%$ \\
\hline Congenital Heart disease & 4 & 50 \\
\hline Cleft palate & 1 & 12.5 \\
\hline Cleft Lip & 1 & 12.5 \\
\hline Open Neural Tube defects & 1 & 12.5 \\
\hline Tracheo-esophageal fistula & 1 & 12.5 \\
\hline
\end{tabular}

\section{DISCUSSION}

GDM is the most common metabolic disorder complicating pregnancy. Approximately 3-5\% of all pregnancies are complicated by diabetes and $90 \%$ of these are GDM and rest is pregestational. Study by Deborah L Conway shows an incidence of 2-5\%. ${ }^{1}$ Our study included 134 GDM patient's incidence being $2.4 \%$. According to Hold $\mathrm{M}$ et al GDM is linked to several maternal, fetal and neonatal complications the characteristics of which are same is that of pregestational diabetes. $^{2}$

Age of study group was between 28 and 40 yrs. They were subjected to $50 \mathrm{gm}$ OGCT at 24-28 weeks gestation as a screening test. Green MF recommends 50gm OGCT as screening test. ${ }^{3}$ If the value is $>=130 \mathrm{mg} / \mathrm{dl} 100 \mathrm{gm}$ oral glucose tolerance test (OGTT) done as a diagnostic test. O Sullivan et al recommends $100 \mathrm{gm} \mathrm{GTT}$ for diagnosis which is the most commonly used one. ${ }^{4}$ One abnormal value was considered as impaired glucose tolerance test and if two or more values abnormal as GDM.

Management options included Medical nutrition therapy (MNT), Insulin therapy and exercise for control of sugar and fetal and maternal monitoring. International workshop on GDM concluded that failed MNT where FBS $>95 \mathrm{mg} / \mathrm{dl}$ and $\mathrm{PPBS}>120 \mathrm{mg} / \mathrm{dl}$ mean glucose
$>105 \mathrm{mg} / \mathrm{dl}$ are indications to start insulin, dose adjusted to maintain FBS $<95 \mathrm{mg} / \mathrm{dl} 2 \mathrm{hr}$ PPBS $<120 \mathrm{mg} / \mathrm{dl} .{ }^{5}$ Langer $O$ et al suggests that Metformin can be used as an alternative therapy. ${ }^{6}$ In our study diet and insulin was given for $64.92 \%$ patients MNT alone in $32.8 \%$ patients and three required diet control, insulin and Metformin.

Majority were between 25-29 years mean age was 27.79. Incidence of GDM was high among age $>=30$ years indicate age is a risk factor for developing GDM. Socioeconomic and educational status does not found to have any influence. Majority were multi gravid. Previous history of GDM, abortions, PIH, macrosomia, NND, IUD, congenital anomalies and family history of DM were there in significant numbers. Four patients had polycystic ovarian disease and 14 had infertility treatment.

USS done in all cases main indication being to $\mathrm{R} / \mathrm{O}$ anomaly and for fetal wellbeing and Doppler done when indicated. According to London $\mathrm{MB}$ et al fetal surveillance should be started at 28-32wks which can eliminate stillbirth, fetal compromise and preterm delivery. ${ }^{7}$ Kofinas A et al in their study recommended Doppler ultrasound study as a clinical tool for fetal surviellance in pregnancy at risk for placental vascular disease. ${ }^{8}$ Majority had normal ultrasonogram, abnormal findings were Hydramnios, Congenital anomalies, macrosomia and FGR. Common maternal problems were UTI, vulvitis PIH, Hydramnios premature rapture of membranes (PROM), preterm labor, abruptio placenta, meconium stained amniotic fluid. In this study, PIH was present in $11.9 \%$ cases. Macrosomia was present in $2.9 \%$ cases. Person B and Hanson U. in a study proved that inspite of proper control of diabetes by close obstetric management there still remains an increased risk of maternal complications commonest being infections, PIH, PROM, hydramnios and short term and long term neonatal morbidity in infant. ${ }^{9}$

Labor was induced around 38 weeks and $16.4 \%$ of patients induced before 38 weeks due to complications like PIH and FGR. No patients allowed going past date. This is because of IUD and still birth increase after 38 weeks of gestation. According to Rasmussen MJ et al delivery before full term is not recommended unless there is evidence of macrosomia, polyhydramnios, FGR, poor diabetic control or other obstetric complications Out of 134 cases, $62.68 \%$ of patients had spontaneous onset of labor and elective termination was done in $37.32 \%$ cases. ${ }^{10}$ Delivery was by vaginal route in $41 \%$ cases. Caesarean rate was $59 \%$ out of these, $22 \%$ elective C.S. and $78 \%$ were emergency C.S. The common indications were previous caesarean section, severe PIH, FGR, Cephalopelvic disproportion (CPD) fetal distress, meconium stained amniotic fluid and failed indication. The Ante-partum, intra-partum and perinatal complications were less in well controlled GDM patients. The mean birth weight in the study group was $2.9 \mathrm{~kg}$, majority were between 2.5 to $2.9 \mathrm{~kg}$. Two (1.49\%) were 
above $4 \mathrm{~kg}$. Over all perinatal morbidity was (9.7\%). $43 \%$ babies were observed in NICU and $25 \%$ needed admission due to complications like jaundice, hypoglycemia RDS and sepsis. Hypoglycemia was the most common perinatal problem. Overall incidence of neonatal hypoglycemia in GDM is about $0.5-4 \%$ in at term appropriate for age infant. Mimouni $\mathrm{F}$ et al found that infants of women with GDM have an incidence of neonatal hypoglycemia about $30-50 \% .{ }^{11}$ In our study hypoglycemia was observed in $13(32.5 \%)$ babies and sepsis in 9 babies. Hyperbilirubinemia was found in 5 babies, meconium aspiration syndrome in 3 babies and respiratory distress syndrome in 2 babies.

Among the $8(3.73 \%)$ babies who had congenital anomalies 4 had congenital heart diseases of which 2 were patent ductus arteriosus. There was 1 case of cleft lip and 1 case of cleft palate. Also, there was 1 case of meningomyelocele and 1 case of tracheoesophageal fistula. Holland Brews reported incidence of congenital anomaly to be about $1-2 \%$ in GDM. ${ }^{12}$ There were 2 cases of IUDs and 2 cases of Neonatal deaths and no still birth. Of the 2 NND 1 was a preterm baby with sepsis and 1 baby had intra cranial hemorrhage with septic shock. In the well-controlled patient's perinatal loss was zero. Perinatal mortality occurred more in uncontrolled or poorly controlled diabetes, still births are usually due to poor control undetected cases or when there is a previous history of IUD or presence of PIH

In most of cases, blood sugar was normal after delivery and 7 cases needed insulin. Maternal morbidity is increased in patients with GDM. There were 7 cases of post-operative wound infection among the 79 LSCS patients. There was 1 case of secondary post-partum hemorrhage and for that patient subtotal hysterectomy was done to control the PPH. There were 9 cases of postoperative pyrexia. Inspite of glycemic control immediately after delivery GDM generally improves but there is a risk of developing Type 2 DM, Hypertension, Cardiovascular disease and obesity. Kjos SL suggests that in patients with GDM, 100 gram GTT should be done 6-8 wks postpartum to R/O DM. ${ }^{13}$

\section{CONCLUSION}

Incidence of GDM in this study was $2.4 \%$. The occurrence of GDM is a high-risk situation. Maternal and perinatal morbidity and mortality is increased in women with GDM. All pregnant women should be screened for gestational diabetes with a $50 \mathrm{gm}$ oral glucose load followed by a glucose determination1hour later. Those with abnormal value should undergo diagnostic test. This helps to detect all GDM cases earlier and so timely intervention can be done which will reduces the complications. Proper control of diabetes periconceptionally reduces incidence of fetal anomalies and glycemic control during the latter half of pregnancy reduces perinatal complications. Intensive sugar control, fetal surveillance, prevention of obstetric complications, timely delivery and intensive neonatal care by a team approach by Obstetrician, Physician preferably Diabetologist and the Neonatologist became the corner stone of management of diabetes in pregnancy. Management includes control of sugar by MNT or insulin, fetal surveillance and timely delivery. Infections, PIH, PROM, Hydramnios and preterm labor were the common maternal complications. Fetal complications include anomalies, macrosomia development of hypoglycemia, respiratory distress, sepsis hypocalcemia, hyperbilirubinaemia and shoulder dystocia and admission to NICU, hypoglycemia being the most common cause. After delivery GDM generally improves but there is a risk of developing Type 2 DM, hypertension, cardiovacular disease and obesity.GTT done 6-8 wks postpartum to R/O DM.

\section{ACKNOWLEDGMENTS}

Authors would like to thank staff members of the Department of OBG TDMCHA in their respective assistance in preparing this manuscript.

\section{Funding: No funding sources}

Conflict of interest: None declared

Ethical approval: The study was approved by the Institutional Ethics Committee University of Kerala

\section{REFERENCES}

1. Deborah C. Management of high risk pregnancy $5^{\text {th }}$ edition; Chapter 20:176-80.

2. Hold M, Merlob P, Friedman S. Gestational diabetes Mellitus; a survey of perinatal complication Diabetes. 1999;40(2):74-8.

3. Greene MF. Screening for gestational diabetes $\mathrm{N}$ Engl J med. 1997;337:1625-6.

4. O'Sullivan JB, Charled D, Mahan CM, Dandrow $\mathrm{RV}$, Gestational diabetes and perinatal mortality rate Am J Obstet Gynecol. 1973;136:901-4.

5. International workshop on gestational diabetesDiabetes care. 1997;20(7);1183-97.

6. Langer O, Berkus M, Brustman L. The rationale for insulin management in gestational diabetes Mellitus Diabetes. 1991;40(2):186.

7. London MB, Langer O, Gabbe SG. Fetal Surveillance in pregnancies complicated by insulin dependent diabetes mellitus. AMJ Obstet Gynecol. 1992;167;617-21.

8. Kofinas A, Perry M, Swain M. Uteroplacental Doppler flow velocimetry wave from analysis. AMJ perinatal. 1991;8;273-7.

9. Person B, Hanson U, Neonatal morbidities in gestational diabetes mellitus. Diabetes. 1998;21(2)142-9.

10. Ramussen MJ, Firth R, Roley M, Stronge JM, The timing of delivery in diabetic pregnancy. Obstet Gynecol. 1992;32;313-31.

11. Mimouni F, Tsang RC, Aertzberg VS. Polycythemia, hypomagnesemia and hypocalcemia in infants of 
diabetic pregnancies. AM J Dis child. 1986;140,798800.

12. Holland B. Manual of Obstetrics; $4^{\text {th }}$ ed. Elsevier;2015:126-37.

13. Kjos SL, Peteers RK, Utility of early post-partum glucose tolerance testing. Diabetes. 1995;44:586-91.
Cite this article as: Sathiamma PK, Lalithambica K. A prospective study on maternal and perinatal outcome of gestational diabetes mellitus. Int J Reprod Contracept Obstet Gynecol 2017;6:2933-8. 